MARIA A. KONSTANTINIDOU, M.Sc. ${ }^{1}$

(Corresponding author)

E-mail: mkonstaa@central.ntua.gr

KONSTANTINOS L. KEPAPTSOGLOU, Ph.D. ${ }^{2}$

E-mail: kkepap@central.ntua.gr

ANTONY STATHOPOULOS, Ph.D. ${ }^{1}$

E-mail: a.stath@transport.ntua.gr

1 Department of Transportation Planning and Engineering

School of Civil Engineering

National Technical University of Athens

9 Iroon Polytechniou Str., GR-15780, Zografou

Campus, Athens, Greece

2 Department of Infrastructure and Rural Development

School of Rural and Surveying Engineering

National Technical University of Athens

9, Iroon Polytechniou Str. GR-15780, Zografou Campus

Athens, Greece
Transport in Emergency Situations

Original Scientific Paper

Submitted: 15 Sep. 2017

Accepted: 21 Sep. 2018

\title{
A MULTI-OBJECTIVE NETWORK DESIGN MODEL FOR POST-DISASTER TRANSPORTATION NETWORK MANAGEMENT
}

\begin{abstract}
Despite their inherent vulnerability to structural and functional degradation, transportation networks play a vital role in the aftermath of disasters by ensuring physical access to the affected communities and providing services according to the generated needs. In this setting of operational conditions and service needs which deviate from normal, a restructuring of network functions is deemed to be beneficial for overall network serviceability. In such context, this paper explores the planning of post-disaster operations on a network following a hazardous event on one of the network's nodes. Lane reversal, demand regulation and path activation are applied to provide an optimally reconfigured network with reallocated demand, so that the network performance is maximized. The problem is formulated as a bi-level optimization model; the upper level determines the optimal network management strategy implementation scheme while the lower level assigns traffic on the network. Three performance indices are used for that purpose: the total network travel time (TNTT), the total network flow (TNF) and the special origin-destination pair (OD pair) accessibility. A genetic algorithm coupled with a traffic assignment process is used as a solution methodology. Application of the model on a real urban network proves the computational efficiency of the algorithm; the model systematically produces robust results of enhanced network performance, indicating its value as an operation planning tool.
\end{abstract}

\section{KEY WORDS}

disasters; network performance; network design model; lane reversal; demand regulation; accessibility; genetic algorithms;

\section{INTRODUCTION}

Impacts of natural and man-made disasters have increased in the recent years. According to the World Bank [1], natural disasters, especially climate change-related ones, exhibit an upward trend in number, magnitude and impacts. In addition, recent events from around the world show that, nowadays, human-induced disasters have evolved from abstract scenarios and unlikely past events to real-life threats to the modern world. It is thus obvious that the necessity of effective countermeasures against different types of disasters is more imperative than ever before.

There are three major differences between natural and man-made disasters. The first one regards the extent of disaster impacts. Natural disasters tend to affect extensive areas [2] and are more complex and variable in shape [3]. Man-made disasters, on the other hand, can be seen as single-source catastrophes which extend to their surrounding area $[2,3]$. The second one refers to the impact magnitude. Natural disasters generally have an even impact over the affected area, while the impact of man-made disasters is more concentrated on the origin node [2]. The third difference concerns the occurrence of timing and progression of the phenomenon. While some of the natural disasters can be anticipated and/or their progression can be monitored, man-made disasters cannot be foreseen despite the existence of any possible information about them [4]. 
In both natural and man-made disasters, transportation networks are of significant practical importance since they (a) provide emergency services on the basis of the generated needs, (b) ensure physical access to the affected regions, and (c) are vulnerable to structural and functional degradation [5]. In their study, Zimmerman et al. [6] highlight the importance of network's availability and capacity in the effectiveness of post-disaster operations while they note that the Federal Highway Administration (FHWA) "recognizes the unique challenges posed by the disaster environment on mobility and the safe and secure movement of people and goods". In this context, identification and employment of appropriate management strategies in the planning process of network operations is deemed to be beneficial for overall network serviceability.

In contrast to the typical evacuation studies where traffic is only heading outbound, this paper examines the general operation of a network in the post-disaster phase with the use of an integrated model. The scenario considered assumes a life-threatening, hazardous event on one of the network's nodes. Such an event could include an explosion or a major fire in a building or in a number of buildings. Applying three different management strategies: (a) lane reversal, (b) demand regulation and (c) path activation, enhancement of network performance is pursued through the minimization of the total network travel time (TNTT) and the maximization of the total network flow (TNF) and the special origin-destination pair (OD pair) accessibility. To the best of the authors' knowledge, it is the first time that accessibility between a set of special OD pairs defined on a network is introduced as a performance measure in a post-disaster management context, and it is also the first time that the TNTT and the TNF are coupled with one another and the special OD pair accessibility to form a combined index. This combined index acts as a multi-aspect measure of performance, catching different parameters of network functionality, and is deemed to provide improved results in terms of transportation network redesign on the basis of the generated needs. The problem is classified as a variant of the mixed network design problem (MNDP) and is formulated in terms of bi-level programming; the upper level determines the optimal network management strategy implementation scheme while the lower level assigns traffic on the network. The proposed model is solved using a meta-heuristic algorithm and is applied on the real urban network of a mid-size city in Greece. According to Menoni [7], the urban space raises the complexity of disaster management due to the high concentration of people, activities, structures and infrastructures. It seems thus reasonable to use a real urban network as a test-bed to the problem at hand.
The next section offers a review of research on disaster management in transportation networks and highlights the motivation and contribution of this paper. Next, the proposed model and solution procedure are presented. The model is then applied to the proposed network and results are presented and discussed. The paper concludes with the findings of the study.

\section{BACKGROUND}

As already explained, the disaster environment poses unique challenges on transportation networks. The impact of a catastrophe in terms of physical and functional degradation of the network's components as well as travelers' behavior, combined with the emerging needs for protection of the population, relief operations and restoration activities, imply that planning for disasters is a multi-aspect process. In this setting, continuation of service provision requires restructuring of network functions; this may include network reconfiguration and/or other management tactics on the basis of the strategies employed and the objectives set.

Both management strategies and system objectives are related to the operations undertaken on the post-disaster network. Indeed, consideration of specific types of operations can potentially have an impact on the measures used to evaluate network performance. For example, in evacuation planning, network performance measures could include network clearance time or total system throughput. Nevertheless, in post-disaster network management those indices may be inappropriate. It is thus important that network performance is expressed in a way that can best describe the actual post-disaster network state while fitting the objectives of the operation plan.

Network performance can generally be estimated on the basis of flow-dependent or flow-independent measures [8]. In a post-disaster environment, it is easier to estimate flow-independent measures since they depend solely on network's physical state and avoid the uncertainties of flow estimations. Chang and Nojima [9] argue that flow-dependent measures are of limited practical significance in a post-disaster environment due to the lack of available data. However, in her study, Chang [10] argues that flow-dependent measures are better in providing insights regarding network performance in cases of sudden network changes, such as those caused by disasters. For example, Ukkusuri and Yushimito [11] dismiss the use of the shortest distance paths as the appropriate measure for performance estimation. In their study, the criticality of network links is assessed by link capacity reductions and user equilibrium (UE) analysis for total network travel time estimation. As already explained, flow-dependent measures are susceptible to the inherently present 
stochasticities of the post-disaster environment; variability of travel times on network links can be magnified in a post-disaster state due to congestion phenomena and travelers manifesting short-term behavior [10,12]. In the present framework, flow-dependent and flow-independent measures are combined to provide an integrated network performance index. For this reason, three general types of performance indices are used: the total network travel time (TNTT), the total network flow (TNF) and the special OD pair accessibility.

The TNTT, defined as the sum of all vehicles' travel times, is a popular performance measure in evacuation studies [13]. Studies employing the TNTT in performance evaluation include those of [13-17]. The TNF, on the other hand, refers to the fraction of the demand satisfied in the post-disaster stage and is directly related to the demand regulation strategy, which will be analyzed in the subsequent paragraph. Finally, accessibility generally refers to the ease of approaching a certain destination [18] and can be a distance-based measure, a time-based measure, or a combination of both. Accessibility can be used in a variety of concepts [19], with different types of classification proposed in the literature [19-21]. When used in a disaster management context, accessibility has, until now, focused on various forms of distance-based measures; in most cases, these are based on minimum distance paths in the pre- and post-disaster network states. Distance-based accessibility approaches the problem from a topological point of view, providing thus estimates of post-disaster node connectivity. This kind of measure is usually weighted by some factor, such as population data (e.g. [22]) or pre-disaster OD data (e.g. $[9,10])$. Time-based accessibility measures, on the other hand, relate accessibility with travel time on the network links. However, the stochasticities related to the post-disaster environment and its impact on travel behavior have generally prevented researchers from using these types of models. In the literature, Bono and Gutiérrez [23] used a distance-based accessibility index while Chang and Nojima [9] estimated the post-disaster performance of an earthquake-raided area with three different measures: total length of network open, and total and area-based accessibility. Later, Chang [10] expanded her previous research by formulating the travel time-based accessibility index. Chen et al. [24] developed an accessibility measure based on a random utility theory while Kondo et al. [25] made use of gravity models. Sohn [26] developed a composite measure of travel distance and traffic volume weighted by population data. Finally, Taylor et al. [27] used three types of measures for regional network performance evaluation and influenced the subsequent work of Taylor and D' Este [28] and Taylor and Susilawati [22].
In addition, several management strategies have been proposed in the literature for post-disaster network planning. However, the problem of emergency traffic management has mostly been treated as a continuous network design problem (CNDP), with demand regulation being the major strategy implemented for enhancing post-disaster network performance (e.g. [12,29]). By definition, demand regulation refers to the imposition of some kind of control over the allowable traffic movements. In lida et al. [12], demand regulation has the form of area regulation, implying the determination of vehicular traffic rates allowed to enter the damaged regions. In Sumalee and Kurauchi [29], demand regulation refers to the fraction of traffic allowed to travel between the OD pairs, while in Daganzo and So [30] and Sisiopiku [31] it has the form of access prohibition in highways, therefore referring to "linear regulation of traffic", according to the definition provided by lida et al. [12]. Expanding the practice of focusing solely on demand regulation in emergency traffic management, Konstantinidou et al. [17] argued in favor of incorporating lane-based strategies in the disaster management framework. Among those strategies, lane reversal is probably the most widely applied. Lane reversal is about shifting the direction of some of the opposing lanes on a roadway segment with the objective of capacity augmentation on the basis of the generated needs. Due to its simplicity and straightforward manner, lane reversal has been applied in many evacuation (e.g. [4,32-35]) and post-disaster network management studies (e.g. $[16,17])$.

In the present study, three management strategies (lane reversal, demand regulation and path activation) are employed to enhance post-disaster network functionality. Due to the nature of the design variables, the problem belongs to the category of mixed network design problems (MNDPs) and is formulated as a bi-level optimization problem.

\section{MODEL FRAMEWORK}

Under emergencies, and despite the challenging external conditions, transportation networks are expected to be adequately functional in order to provide vital services for population safety, community restoration and continuation of activities. In order to do so, physical and functional degradation of transportation infrastructure has to be mitigated through careful planning; disaster management is a multi-stage process which begins with pre-disaster planning and system improvement, and extends to post-disaster system response, recovery and reconstruction [36]. The two stages act in a synergetic manner; pre-disaster planning involves strategic decision-making for risk assessment and management, infrastructure improvements to reduce vulnerability and formulation of emergency plans [5]. The post-disaster stage involves tactical and 
operational decision-making for providing critical emergency, recovery and reconstruction services to support society [5]. In both phases, the effective deployment of appropriate management strategies lies at the core of every successful disaster management plan.

In the present study, a hazardous event on one of the network's nodes sets the disaster environment where the problem unfolds. Due to the possible risk of human life losses and injuries, the four city blocks adjacent to the attacked node are blocked as a matter of precaution, setting the capacity of the respective links to zero. The surviving network now has to face: (a) the regular transportation needs of the network users under operational conditions which deviate from normal, (b) the danger that the expected irregularity of traffic patterns may lead to congestion phenomena and gridlocks, and (c) the fact that access to some network nodes may be of particular importance during the post-disaster phase and should be especially considered in an estimation of network serviceability. Such nodes could correspond to facilities which are vital for population safety, community restoration and continuation of activities such as hospitals, police and fire stations, shelters, and so on. It seems thus reasonable that the state of connectivity conditions to/from these nodes should be especially accounted for and evaluated when formulating a disaster management plan. For this reason, in the present problem, a set of three high importance nodes is defined on the network; each of these nodes is assumed to correspond to a facility which is vital for the restoration of the community and its activities in the aftermath of the disaster. Next, a set of special OD pairs is defined; the sets are formulated between the aforementioned network's high importance nodes and other network nodes. In this problem, and considering network size, seven special OD pairs are defined. Each special OD pair is then assumed to be connected by a set of paths determined with the use of a k-shortest path algorithm ( $\mathrm{k}$ equals two). It must be noted that the problem formulation is not restrictive with respect to the number of special OD pairs defined on the network; that is, consideration of the seven special OD pairs in this particular case study is a problem hypothesis in order to test the formulated model.

During the formulation of the disaster management plan, three management strategies are employed on the post-disaster network for the enhancement of its performance: (a) lane reversal, (b) demand regulation, and (c) activation of a set of paths between the network's special OD pairs. Lane reversal attempts to reallocate the available roadway capacity on the basis of the generated needs by shifting the direction of some of the opposing lanes on the roadway segments. Demand regulation aims at managing the demand so that it does not contribute to further deterioration of the network functionality and refers to the fraction of the demand allowed to travel between the network's OD pairs. Finally, in path activation, the algorithm internally seeks to activate the shortest distance and shortest travel time paths between at least four out of the seven special OD pairs defined on the post-disaster network. Performance of the activated paths is then assessed in terms of accessibility. This way, the algorithm puts special weight on the state of connectivity conditions between the network's special OD pairs in the estimation of overall network serviceability. Therefore, access to network's high importance nodes is emphasized and especially accounted for in the formulation of the final network management strategy implementation scheme.

Due to the nature of the design variables (lane reversal and path activation are discrete variables, whereas demand regulation is continuous), the problem at hand belongs to the category of MNDPs and is formulated in terms of bi-level programming. The upper level determines the optimal network management strategy implementation scheme while the lower level assigns traffic on the network. The employment of the three management strategies aims at the formulation of the reconfigured network with reallocated demand so that network performance is maximized.

Three performance indices are used for that purpose: the total network travel time (TNTT), the total network flow (TNF) and the special OD pair accessibility. These three indices act as a multi-aspect measure of performance, catching different parameters of the network function. More specifically, the TNTT serves as a time-based criterion of network performance, which can account for the impact of network's physical deterioration, as well as for the possible variations of travel patterns in a post-disaster setting. Indeed, according to the definition, TNTT is the sum of all vehicle travel times $\left(\sum_{(i, j) \in A}\left(x_{i j} \cdot t_{i j}\right)\right)$, with $x_{i j}$ and $t_{i j}$ being the flow and travel time on link $(i, j)$ respectively, and $A$ being an ordered set of arcs. From this equation, it is obvious that fluctuations of the flow, as well as variations of travel times on the network's links, can result in different values of TNTT. For example, for two links $a$ and $b$ with flows $x_{a}=10$ and $x_{b}=20$, and travel times $t_{a}=5$ and $t_{b}=3$, an increase of travel times to $t_{a}^{\prime}=6$ and $t_{b}^{\prime}=5$ will result in an increase of the TNTT by $45.45 \%$. In addition, the TNF, calculated as the fraction of the demand allowed to travel between the OD pairs in the post-disaster stage, gives additional information about network functionality and helps to gain more insight into the user's perspective. Indeed, the size of the satisfied demand is an indicator of the users' needs satisfaction degree. For example, let the initial demand between the OD pair $(r, s)$ be $q^{r s}=100$. In the aftermath of a disaster, the damaged transportation 


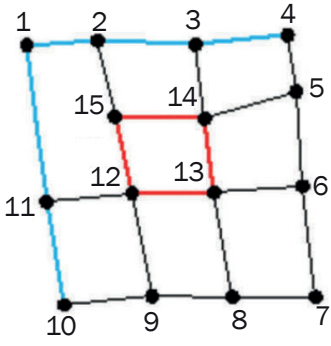

Path P1

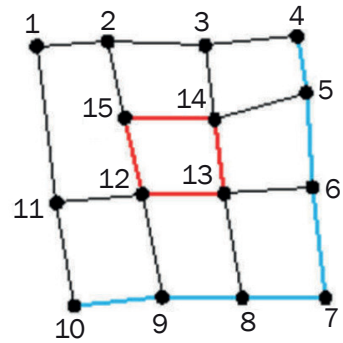

Path P2

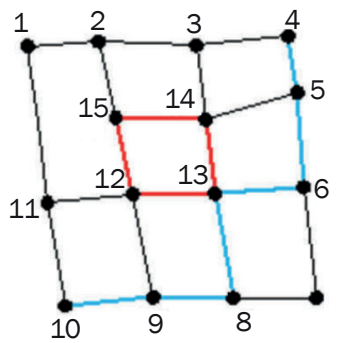

Path P3

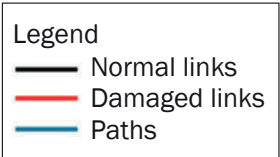

Figure 1 - Illustrative example of path activation infrastructures may not be able to sufficiently serve the total amount of the demand, and the authorities may thereby decide to allow only a percentage (e.g. one half) of the demand to travel. In that case, the other half, that is prohibited from traveling, will remain unsatisfied. Finally, the special OD pair accessibility is a combination of the time-based measure and the distance-based measure, and it is formulated on the basis of the indices developed earlier by Chang and Nojima [9], Chang [10] and Sohn [26]. As already explained, in this model, the accessibility term assesses the state of connectivity conditions between the network's special OD pairs, emphasizing the importance of ensuring, to the extent possible, unobstructed access to network's high importance nodes. This is achieved by the activation of the shortest distance and the shortest travel time paths connecting at least four out of the seven special OD pairs defined on the post-disaster network. It must be once again noted that the accessibility term refers only to the seven special OD pairs defined on the network and to the specific paths (determined with the use of k-shortest path algorithm) connecting them, and not to the whole set of the network's OD pairs. Consideration of the latter is excluded by setting $w^{r s}=0$ in the case $r$ does not belong to the set of high importance nodes $N_{s p}$ (Equation 5) (with $w^{r s}$ being an indicator of the OD pair $(r, s)$ belonging to the special OD pairs defined on the network ( $w^{r s}=0$ or 1$)$ ). For example, in the network illustrated in Figure 1 with four damaged links, let node 4 be the network's only high importance node, and the OD pair $(4,10)$ the network's only special OD pair. In that case, it will be $w^{4,10}=1$ and $w^{r s}=0$ for every other OD pair. If three paths connecting the OD pair $(4,10)$ are defined on the network (paths P1, P2, P3), then the path exhibiting the shortest distance and the shortest travel time will be the one chosen for activation by the algorithm.

The problem follows an iterative solution process. The management strategy implementation scheme derived at each iteration is assessed in terms of the three distinct performance indicators. Optimality is reached when no other implementation scheme can achieve improved network performance.

\subsection{Model formulation}

\subsubsection{Network representation and notation}

Let $G(N, A)$ be a directed network, where $N$ is a set of nodes and $A$ is a set of arcs, with members of $N$ and $A$ respectively formulating ordered pairs within the same set. For each directed arc $i \rightarrow j$, there is a length $d_{i j}$, a free flow travel time $t_{f, i j}$, a capacity $c_{i j}$ and an initial number of lanes $l_{i j}$. Also, let $N_{1} \subseteq N$ be a subset of nodes being the network's centroids. For two centroids $(r, s) \in N_{1}$, the corresponding origin-destination (OD) flow is denoted as $q^{r s}$ and the associated OD matrix as $O D=\left\{q^{r s}\right\}, \forall(r, s) \in N_{1}$. With respect to network assignment, flow and travel time per link are defined as $x_{i j}$ and $t_{i j}$, respectively. Also, let $K_{s p}$ be a set of paths connecting the high importance nodes of subset $N_{s p}$ with specific nodes of subset $N_{1}, K$ a set of paths between centroids $(r, s) \in N_{1}$, and $k \in K$ a single path between $r$ and $s$. In the model, $d_{k}^{r s}, t_{k}^{r s}$, and $f_{k}^{r s}$ are the length of, travel time on, and flow on path $k$ between $r$ and $s$ respectively, while $w^{r s}=1$, if node $r$ belongs to the $N_{s p}$. In addition, $\delta_{i j, k}^{r s}=\{0,1\}$ is defined, with $\delta_{i j, k}^{r s}=1$ if link $i \rightarrow j$ is a part of path $k$. $Y$ and $Z$ are the objective functions for the upper and lower level, respectively. Symbols with an $(*)$ refer to the network before the optimization process.

The design focus of the problem is: (a) the redistribution of lanes along links, (b) the adjustment of the demand between the network's OD pairs, and (c) the activation of the optimal set of paths between the network's special OD pairs. In this context, $y_{i j}$ is the number of lanes along each directed arc $i \rightarrow j$ after the optimization process (whereas $l_{i j}$ is the initial number of lanes before the optimization process), $\varphi^{r s}$ is the demand adjustment rate between the OD pair $(r, s)$, and $\delta_{k}^{r S}=\{0,1\}$ is an indicator of path activation, with path $k$ being activated if $\delta_{k}^{r s}=1$. The sets and variables used in the model are listed below:

$$
\begin{array}{ll}
\text { Sets } & \\
N & \text { - set of nodes } \\
N_{1} & \text { - set of network's centroids } \\
N_{s p} & \text { - set of network's special importance nodes } \\
A & \text { - set of arcs }
\end{array}
$$


$K \quad$ - set of network's paths

$K_{s p} \quad$ - set of paths connecting the special importance nodes of $N_{s p}$ with specific nodes from $N_{1}$

Input variables

$d_{i j} \quad$ - length of link $i \rightarrow j$

$l_{i j} \quad$ - initial number of lanes on link $i \rightarrow j$ (before the optimization process)

$t_{f, i j} \quad$ - free flow travel time on link $i \rightarrow j$

$q^{r s} \quad$ origin-destination flow between the OD pair $(r, s)$

$w^{r s} \quad$ - indicator of the OD pair $(r, s)$ belonging to the special OD pairs defined on the network $\left(w^{r S}=0\right.$ or 1$)$

$\delta_{i j, k}^{r s} \quad$ - indicator of link $i \rightarrow j$ between the OD pair $(r, s)$ being part of path $k\left(\delta_{i j, k}^{r s}=0\right.$ or 1$)$

$\delta_{i j, k}^{r s}{ }^{*} \quad$ - indicator of link $i \rightarrow j$ between the OD pair $(r, s)$ being part of path $\mathrm{k}$ (before the optimization process) $\left(\delta_{k}^{r s^{*}}=0\right.$ or 1$)$

$m_{2}, m_{3}$ - BPR function parameters

\section{Output variables}

$c_{i j} \quad$ - capacity of link $i \rightarrow j$

$x_{i j} \quad$ - flow on link $i \rightarrow j$

$t_{i j} \quad$ - travel time on link $i \rightarrow j$

$t_{i j}^{*} \quad$ - travel time on link $i \rightarrow j$ (before the optimization process)

$f_{k}^{r s} \quad$ - flow on path $k$ between the OD pair $(r, s)$

$d_{k}^{r s} \quad$ - length of path $k$ connecting the OD pair $(r, s)$

$d_{k}^{r r^{*}} \quad$ - length of path $k$ connecting the OD pair $(r, s)$ (before the optimization process)

$t_{k}^{r s} \quad$ - travel time on path $k$ connecting the OD pair $(r, s)$

$t_{k}^{r s^{*}} \quad$ - travel time on path $k$ connecting the OD pair $(r, s)$ (before the optimization process)

\section{Decision variables}

$y_{i j} \quad$ - number of lanes on link $i \rightarrow j$ (after the optimization process)

$\varphi^{r s} \quad$ - demand adjustment rate between the OD pair $(r, s)$

$\delta_{k}^{r s} \quad$ - indicator of path $\mathrm{k}$ between the OD pair $(r, s)$ being activated $\left(\delta_{k}^{r s}=0\right.$ or 1$)$

The problem is formulated as a bi-level mathematical programming model; the upper level determines the optimal network management strategy implementation scheme while the lower level assigns traffic on the network. In this model, traffic assignment follows the Wardrop's first principle of the user equilibrium (UE). The problem follows an iterative solution process. First, the algorithm assumes the decision variables of the problem to take some values within their valid range. Link travel times and link flows are then calculated from the lower level problem and transferred to the upper level problem as input. Next, the objective function value is derived and assessed in terms of the three performance indicators and the process is repeated. Optimality is reached when additional iterations cannot improve network performance any further.

\subsubsection{Bi-level network optimization model}

The upper level optimization problem is as follows:

$$
\begin{aligned}
\min Y= & \left\|\sum_{(i, j) \in A} x_{i j}\left(\varphi^{r s}\right) \cdot t_{i j}\left(x_{i j}\right)\right\|-\left\|\sum_{r \in N_{1}} \sum_{s \in N_{1}} \varphi^{r s} \cdot q^{r s}\right\| \\
& +\|\left[\sum_{r \in N_{s p}} \sum_{s \in N_{1}} \sum_{k \in K_{s p}} \delta_{k}^{r s} \cdot w^{r s} \cdot\left(\frac{d_{k}^{r s}}{d_{k}^{s *}}\right)\right. \\
& \left.+\sum_{r \in N_{s p}} \sum_{s \in N_{1}} \sum_{k \in K_{s p}} \delta_{k}^{r s} \cdot w^{r s} \cdot\left(\frac{t_{k}^{r s}}{t_{k}^{r s *}}\right)\right] \|
\end{aligned}
$$

subject to:

$$
\begin{aligned}
& y_{i j}=l_{i j}+l_{j i}-y_{j i} \quad \forall(i, j) \in A \\
& y_{i j} \in Z \quad \forall(i, j) \in A \\
& c_{i j}=c_{i j}\left(y_{i j}\right) \quad \forall(i, j) \in A \\
& w^{r s}=\left\{\begin{array}{l}
1, \text { if } r \in N_{s p} \\
0, \text { otherwise }
\end{array} s \in N_{1}\right. \\
& d_{k}^{r s}=\sum_{i \neq j} d_{i j} \cdot \delta_{i j, k}^{r s} \quad \forall(i, j) \in A, k \in K_{s p}, r \in N_{s p}, s \in N_{1} \\
& t_{k}^{r s}=\sum_{i \neq j} t_{i j} \cdot \delta_{i j, k}^{r s} \quad \forall(i, j) \in A, k \in K_{s p}, r \in N_{s p}, s \in N_{1} \\
& \begin{aligned}
\delta_{i j, k}^{r s}=\left\{\begin{array}{l}
1, \text { if link } i \rightarrow j \text { belongs to path } k \\
0, \text { otherwise }
\end{array}\right. \\
\forall(i, j) \in A, k \in K_{s p}, r \in N_{s p, s \in N}
\end{aligned} \\
& d_{k}^{r s *}=\sum_{i \neq j} d_{i j} \cdot \delta_{i j, k}^{r s *} \quad \forall(i, j) \in A, k \in K_{s p}, r \in N_{s p}, s \in N_{1} \\
& t_{k}^{r s^{*}}=\sum_{i \neq j} t_{i j}^{*} \cdot \delta_{i j, k}^{r * *} \quad \forall(i, j) \in A, k \in K_{s p}, r \in N_{s p}, s \in N_{1} \\
& \begin{aligned}
\delta_{i j, k}^{r * *}=\left\{\begin{array}{l}
1, \text { if link } i \rightarrow j \text { belongs to path } k \\
0, \text { otherwise }
\end{array}\right. \\
\forall(i, j) \in A, k \in K_{s p}, r \in N_{s p}, s \in N_{1}
\end{aligned}
\end{aligned}
$$

The lower level UE traffic assignment problem is formulated as follows:

$$
\min Z=\sum_{(i, j) \in A} \int_{0}^{x_{i j}} t_{i j}\left(x_{i j}\right) d x
$$

subject to:

$$
\begin{aligned}
\sum_{k \in K} f_{k}^{r s}=\varphi^{r s} \cdot q^{r s} \quad \forall k \in K, \forall(r, s) \in N_{1} \\
f_{k}^{r s} \geq 0 \quad \forall k \in K, \forall(r, s) \in N_{1} \\
x_{i j}=\sum_{r} \sum_{s} \sum_{k} f_{k}^{r s} \cdot \delta_{i j, k}^{r s} \\
\quad \forall(i, j) \in A, \forall k \in K, \forall(r, s) \in N_{1} \\
x_{i j} \geq 0 \quad \forall(i, j) \in A,
\end{aligned}
$$




$$
t_{i j}=t_{f, i j} \cdot\left(1+m_{2} \cdot\left(\frac{x_{i j}}{c_{i j}}\right)^{m 3}\right) \quad \forall(i, j) \in A
$$

Equation 1 corresponds to the upper-level objective function and consists of three parts: minimization of the TNTT, maximization of the TNF and maximization of the special OD pair accessibility. Maximization of accessibility, i.e., maximization of the ease of approaching a certain destination, goes through the minimization of the distance traveled and the travel time spent in order to reach that destination. Therefore, maximization of the special OD pair accessibility is achieved through the activation of those paths between the network's special OD pairs which exhibit the shortest path lengths and the shortest path travel times. This is the reason why, for the special OD pair accessibility to be maximized, the respective term, defined through path lengths and path travel times, must yield a minimum. All terms are normalized $(\|\|)$, with the method described in Section 3.2.3. Equation 2 defines the number of lanes per directional link. Equation 3 restricts the decision variable to be an integer. Equation 4 sets link capacity. Equation 5 is an indicator of node importance. Equations 6 and 9 calculate path lengths on the optimized and non-optimized networks respectively, while the same applies to Equations 7 and 10 with respect to path travel times. Equations 8 and 11 are indicators of a link belonging to the path between the OD pair on the optimized and non-optimized networks, respectively. Equations 12-17 correspond to the lower-level UE traffic assignment process with Equation 17 being the Bureau of Public Roads (BPR) function. Traffic assignment on the optimized network follows the typical process. At each iteration, new input data, derived from the use of a genetic algorithm and corresponding to capacity per direction changes (number of lanes per directional link) as well as adjustment of the other decision variables' values, are fed to the lower level problem and network assignment is repeated.

\subsubsection{Objective function normalization}

In order that the results of the optimization process are not affected by the different measurement units and orders of magnitude of the three objective function components $Z_{k}$, the latter are transformed into dimensionless, normalized terms $Z_{k, n o r m}$ according to the method of Proos et al. [37]:

$$
Z_{k, \text { norm }}=\frac{Z_{k}}{\left|Z_{k, \max }\right|}
$$

where $\left|Z_{k, \max }\right|$ is the maximum possible value of $Z_{k}$ without constraint violations, and $Z_{k, n o r m}$ lies in the $[0,1]$ interval. The maximum values for each objective function component were calculated as follows:

TNTT: TNTT derived from the assignment of traffic on the non-optimized, post-disaster network is used as the maximum value of this objective function component. This is reasonable, since the TNTT value of the non-optimized, post-disaster network will certainly be larger than the ones derived after the employment of the three management strategies.

TNF: The initial, non-adjusted value of total network demand of the pre-disaster network is used as the upper bound in this case. Due to the nature of the demand regulation strategy (i.e., imposition of demand adjustment rates between the OD pairs so that the demand does not overwhelm the damaged transportation infrastructures), each TNF value derived from the optimization process will most likely be lower, and not in any case larger, than the initial TNF value of the pre-disaster network.

The special OD pair accessibility: Distances and travel times of the paths connecting the network's special OD pairs on the non-optimized, post-disaster network are equal to the maximum values used. As already explained, maximization of accessibility to a destination is achieved through the minimization of the distance traveled and the travel time spent in order to reach that destination. Therefore, maximization of the special OD pair accessibility will be achieved by activation of the shortest distance and the shortest travel time paths connecting them. It is thus obvious that path distances and path travel times derived between those pairs on the non-optimized, post-disaster network will always be larger than the respective ones achieved on the optimized network.

\subsection{Solution method}

The discrete variables involved in the MNDPs and their bi-level nature make these problems NP-hard and non-convex so that one cannot resort to exact algorithms to solve them [38,39]. Therefore, a genetic algorithm (GA) coupled with a traffic assignment process is used as a solution methodology. GAs are known to produce robust results within reasonable execution time and may be the only option available in combinatorial problems due to the exponential time required to reach a solution [40].

The problem is formulated on the basis of three design variables: (a) $y_{i j}$, which regulates lane redistribution along each directed link $i \rightarrow j$, (b) $\varphi^{r s}$, which adjusts the demand between each OD pair $(r, s)$, and (c) $\delta_{k}^{r s}$, which activates path $\mathrm{k}$ between the special OD pair $(r, s)$. Thus, representation of a candidate solution is made possible by three strings:

$\left[y_{12} \ldots y_{i j} \ldots\right] \quad i<j, \forall(i, j) \in A$

$\left[\varphi^{12} \ldots \varphi^{r s} \ldots\right] \quad r<s, \forall(r, s) \in N_{1}$

$\left[\delta_{1}^{12} \ldots \delta_{k}^{12} \ldots \delta_{1}^{r s} \ldots \delta_{k}^{r s} \ldots\right] \quad r \in N_{s p}, s \in N_{1}, k \in K_{s p}$

where: 


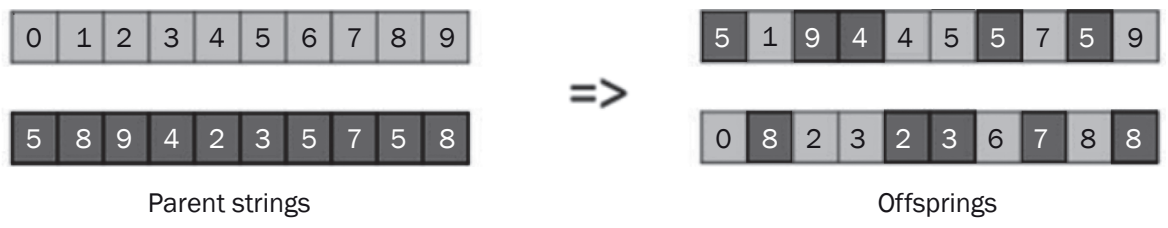

Figure 2 - Uniform crossover with fixed probability of 0.5 [41]
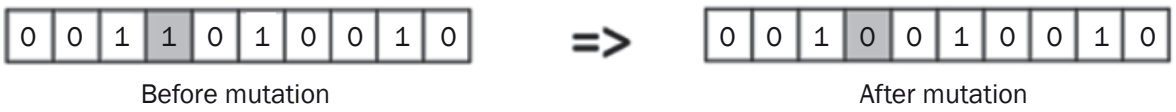

Figure 3 - Random mutation on a binary variable [42]

$$
\begin{aligned}
& y_{i j} \in Z \quad i<j, \forall(i, j) \in A \\
& 0 \leq \varphi^{r s} \leq 1 \quad r<s, \forall(r, s) \in N_{1} \\
& \delta_{k}^{r s}=\left\{\begin{array}{l}
1, \text { if path } k \text { is activated } \\
0, \text { otherwise }
\end{array} \quad r \in N_{s p}, s \in N_{1}, k \in K_{s p}\right.
\end{aligned}
$$

Each string is manipulated separately by the algorithm's genetic operators. The uniform crossover method, in which new candidate solutions are generated by the exchange with a fixed probability of genes between the parent strings and crossover points are randomly chosen, has been used. Figure 2 shows an example of the uniform crossover method with a fixed probability of 0.5 . In this case, each new generated string (offspring) will have half of its genes from the first parent and the other half from the second one. As far as the mutation is concerned, the mutated gene is replaced by a randomly generated number within its valid range. Figure 3 provides an illustrative example.

Equation 1 acts as the fitness measure of the GA with its values obtained through: (a) variables $\varphi^{r s}$ and $\delta_{k}^{r s}$, which are introduced directly into Equation 1, (b) variable $y_{i j}$, which is calculated through Equation 2, and (c) the lower level traffic assignment problem. It must be noted that lane redistribution along a link is treated as a hard constraint and non-feasible candidate solutions are discarded. Also, the algorithm requires that there is a path activated between at least four out of the seven special OD pairs defined on the network. The GA is terminated after pre-specified running time.

\section{APPLICATION}

\subsection{Overview, assumptions and scenario}

The proposed model is applied on the urban roadway network of a mid-size city in Greece (Figure 4). The network consists of 303 bi-directional links, 291 of them having one lane per direction, while the other 12 have two lanes per direction. The analysis assumes a capacity of 900 veh/lane and 1,000 veh/lane as well as speed limits of $50 \mathrm{~km} / \mathrm{h}$ and $70 \mathrm{~km} / \mathrm{h}$ respectively on the network's links. When no travel lanes are available per direction, free flow travel time is assumed to be a very large number (e.g. 99,999).

A hazard crisis scenario is assumed to set the disaster environment where the problem unfolds. More specifically, a life-threatening, hazardous event (e.g. an explosion or a major fire in a building or in a number of buildings) on node 844 leads to the precautionary blockage of the four city blocks adjacent to the attacked node and their respective links (Figure 3). As a result, 14 links are closed to traffic, and their respective capacity is set to zero. Traffic is then assigned to the rest of the network. A peak hour OD matrix of 6,649 vehicle trips is used. In addition, three high importance nodes (nodes 801, 863, and 898) and a set of seven special OD pairs (pairs 890-801, 863-801, 863-905, 874-898, 869-898, 871-898, and 872-898) are defined, with the respective paths between those pairs being identified with the use of a $k$-shortest path algorithm ( $k$ equals two). The model then requires that, on the redesigned network, there is a shortest distance and a shortest travel time path activated between at least four, out of seven, special OD pairs defined; otherwise, the solution is discarded.

\subsection{GA analyses and performance}

One population (POP) size (100), three crossover rate $(C R)$ values $(0.4,0.6$, and 0.8$)$ and three mutation rate $(M R)$ values $(0.05,0.10$, and 0.15$)$ were selected and their combinations in multiple sets of triplets (sets of $1 \cdot 3 \cdot 3=9$ experiments) were tested on an Intel $i 7^{\mathrm{TM}}$ processor with $4 \mathrm{~GB}$ of RAM. Fifteen runs were executed for each triplet as each experiment was executed for $2 \mathrm{~h}$. Table 1 presents the results (best run of all sets and average of all runs) in terms of objective function (OF) value minimization.

There is a discrepancy in the results regarding the optimization of the OF value and its individual components; this is because the objective function consists of three terms whose optimization process does not follow the same trend. More specifically, while the optimization process of the TNTT and the special OD pair 


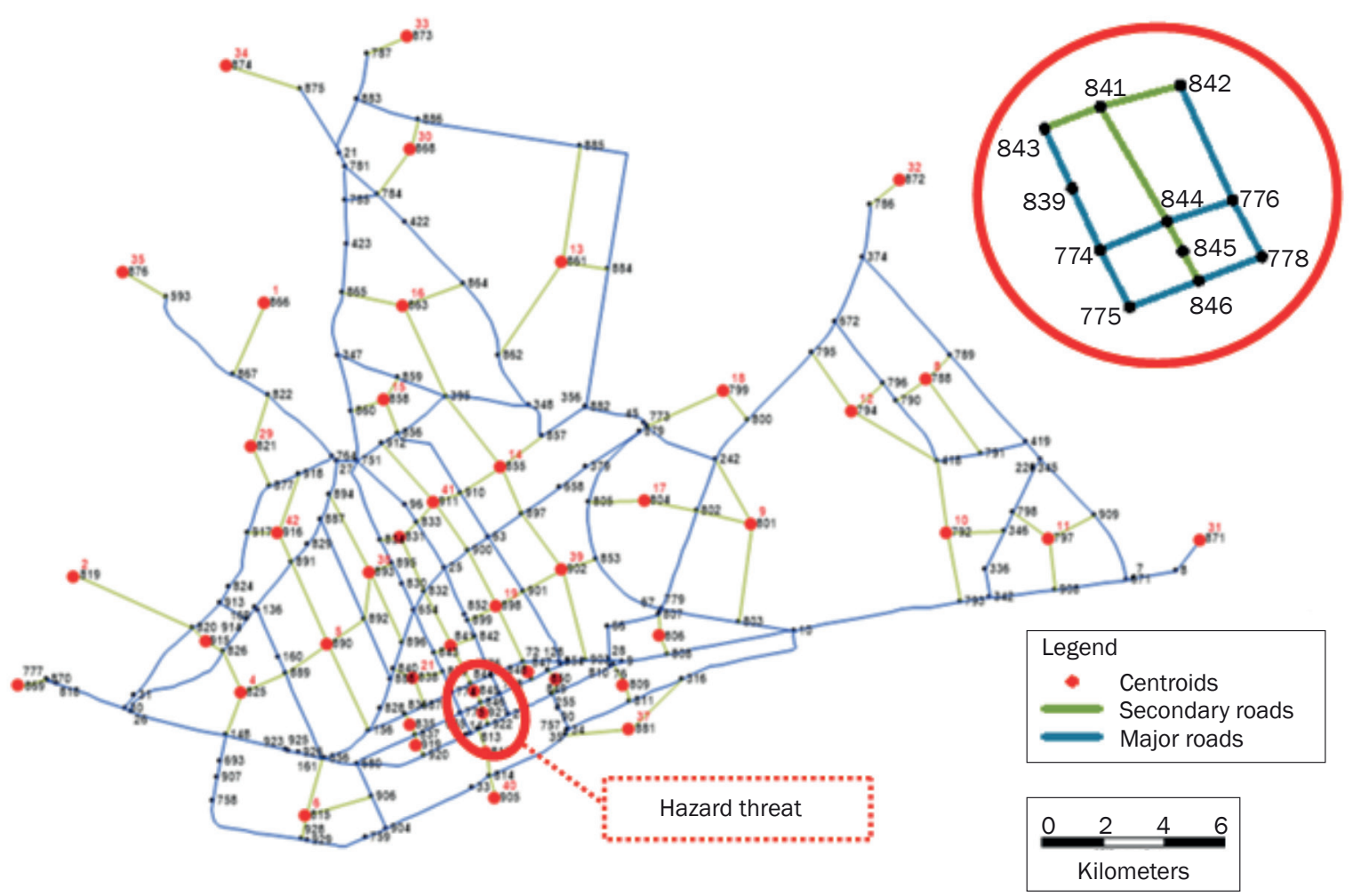

Figure 4 - The urban roadway network of a mid-size city in Greece

Table 1 - Best results for objective function minimization

\begin{tabular}{|c|c|c|c|c|c|c|c|}
\hline & & & \multicolumn{4}{|c|}{ Objective function best runs } & \multirow{2}{*}{$\begin{array}{l}\text { Objective } \\
\text { function } \\
\text { (Average of } \\
\text { all runs) }\end{array}$} \\
\hline & & & $\begin{array}{l}\text { Total network } \\
\text { travel time } \\
\text { [veh hours] }\end{array}$ & $\begin{array}{c}\text { Total network } \\
\text { flow } \\
\text { [veh trips] }\end{array}$ & $\begin{array}{l}\text { Special OD-Pair } \\
\text { accessibility }\end{array}$ & $\begin{array}{l}\text { Objective } \\
\text { function }\end{array}$ & \\
\hline \multicolumn{8}{|c|}{ Experiment combination } \\
\hline POP & $\mathrm{CR}$ & MR & & & & & \\
\hline 100 & 0.4 & 0.05 & 0.109654 & 0.829975 & 0.200029 & -0.520292 & -0.221572 \\
\hline 100 & 0.4 & 0.10 & 0.150885 & 0.832305 & 0.200326 & -0.481093 & -0.385912 \\
\hline 100 & 0.4 & 0.15 & 0.125429 & 0.841549 & 0.206893 & -0.509228 & -0.187915 \\
\hline 100 & 0.6 & 0.05 & 0.172618 & 0.854628 & 0.200000 & -0.482010 & -0.213195 \\
\hline 100 & 0.6 & 0.10 & 0.153996 & 0.849018 & 0.202828 & -0.492194 & -0.195787 \\
\hline 100 & 0.6 & 0.15 & 0.117192 & 0.856237 & 0.200001 & -0.539045 & -0.255338 \\
\hline 100 & 0.8 & 0.05 & 0.162310 & 0.861781 & 0.200055 & -0.499416 & -0.346264 \\
\hline 100 & 0.8 & 0.10 & 0.170382 & 0.853363 & 0.207646 & -0.475335 & -0.355990 \\
\hline 100 & 0.8 & 0.15 & 0.102357 & 0.857789 & 0.200088 & -0.555344 & -0.328316 \\
\hline \multicolumn{3}{|l|}{ Average } & 0.140536 & 0.848516 & 0.201985 & -0.505995 & -0.276699 \\
\hline \multicolumn{3}{|c|}{ Standard deviation } & 0.027081 & 0.011390 & 0.003135 & 0.027640 & 0.077197 \\
\hline \multicolumn{3}{|c|}{ Coefficient of variation [\%] } & 19.269669 & 1.342298 & 1.552083 & 5.462425 & 27.899410 \\
\hline \multicolumn{3}{|c|}{ Best experiment } & 0.102357 & 0.857789 & 0.200088 & -0.555344 & na \\
\hline \multicolumn{3}{|c|}{ Deviation from average [\%] } & 27.166365 & 1.092788 & 0.939458 & 9.752761 & na \\
\hline
\end{tabular}

na: not applicable 
Konstantinidou MA, Kepaptsoglou KL, Stathopoulos A. A Multi-objective Network Design Model for Post-disaster Transportation Network...

Table 2 - Best results for objective function minimization for different speed limits

\begin{tabular}{||l|c|c|c|c||}
\hline \multirow{2}{*}{} & \multicolumn{4}{|c||}{ Objective function best runs } \\
\cline { 2 - 5 } & $\begin{array}{c}\text { Total network } \\
\text { travel time } \\
\text { [veh hours] }\end{array}$ & $\begin{array}{c}\text { Total network flow } \\
\text { [veh trips] }\end{array}$ & $\begin{array}{c}\text { Special OD pair } \\
\text { accessibility }\end{array}$ & Objective function \\
\hline \hline \multicolumn{5}{|c|}{ Speed limits: $v=60 \mathrm{~km} / \mathrm{h}$ and $80 \mathrm{~km} / \mathrm{h}$} \\
\hline Average & 0.131255 & 0.857640 & 0.192231 & -0.534154 \\
\hline Standard deviation & 0.028715 & 0.010758 & 0.003268 & 0.027143 \\
\hline Coefficient of variation [\%] & 21.877140 & 1.254343 & 1.699932 & 5.081578 \\
\hline \hline \multicolumn{5}{|c|}{ Speed limits: $v=40 \mathrm{~km} / \mathrm{h}$ and $60 \mathrm{~km} / \mathrm{h}$} \\
\hline Average & 0.184679 & 0.829304 & 0.214245 & -0.430380 \\
\hline Standard deviation & 0.023563 & 0.012820 & 0.003043 & 0.036770 \\
\hline Coefficient of variation [\%] & 12.758915 & 1.545851 & 1.420160 & 8.543569 \\
\hline
\end{tabular}

accessibility terms as individual indices would yield a minimum (since the maximization of the ease of approaching a certain destination (i.e., accessibility) is achieved through the minimization of the distance traveled and the travel time spent in order to reach that destination, and thus the activation of the shortest distance and shortest travel time paths between the network's special OD pairs), the optimization of the TNF would yield a maximum. That is, the TNTT and the special OD pair accessibility components act competitively to the TNF component when merged in a combined index.

The minimum average OF value is $(-0.385912)$ for the combination where $\mathrm{POP}=100, \mathrm{CR}=0.4, \mathrm{MR}=0.1$ while the average OF value for the best run experiments is $(-0.328316)$ (combination where $P O P=100$, $\mathrm{CR}=0.8, \mathrm{MR}=0.15)$. The average OF value for the best runs is $(-0.505995)$. The coefficient of variation is low (5.46\%), indicating the efficiency of the GA in producing results of similar quality. The variation coefficients for the individual terms of the objective function range from $1.34 \%$ and $1.55 \%$ for the TNF and the special OD pair accessibility components respectively, to $19.27 \%$ for the TNTT component. More specifically, in all experiments, the algorithm shows a remarkable robustness in finding the optimal demand adjustment rates between the OD pairs as well as the shortest distance and the shortest travel time paths between the special OD pairs, thus maximizing the TNF and the special OD pair accessibility components, respectively. The maximum TNF value is 0.861781 for the experiment where $\mathrm{POP}=100, \mathrm{CR}=0.8, \mathrm{MR}=0.05$, while the best special $\mathrm{OD}$ pair accessibility value is 0.2 for the experiment where $P O P=100, C R=0.6, M R=0.05$. Both values lie very close to the achieved ones of the best run the experiment where $\mathrm{POP}=100, \mathrm{CR}=0.8, \mathrm{MR}=0.15$ (for which there are 946 trips lost and 42 lanes reversed). However, the TNTT results exhibit a greater variability in their values. The minimum TNTT value is $(0.102357)$ of the best run experiment, where $\mathrm{POP}=100, \mathrm{CR}=0.8$,
$\mathrm{MR}=0.15$, while the next two lowest values are 0.109654 and 0.117192 for the experiments where $\mathrm{POP}=100, \mathrm{CR}=0.4, \mathrm{MR}=0.05$, and $\mathrm{POP}=100, \mathrm{CR}=0.6$, $M R=0.15$, respectively. Those three experiments are also the ones exhibiting the lowest OF values; this fact implies that the optimization process is mainly driven by the TNTT component since the TNF and the special OD pair accessibility values remain almost steady in all experiments.

The analyses performed do not show a clear trend regarding the impact of different crossover rates on the best run experiment results. However, it seems that higher mutation rate experiments have a slightly better performance. The opposite is valid for the average of all runs OF values. In this case it seems that higher crossover values have better performance, while the role of the mutation rate values is not evident.

The computational efficiency of the algorithm is also demonstrated by an additional set of analyses performed for a running time of $2 \mathrm{~h}$, taking into account different speed limits on the network's links (Table 2). Combinations of $60 \mathrm{~km} / \mathrm{h}$ and $80 \mathrm{~km} / \mathrm{h}$, as well as 40 $\mathrm{km} / \mathrm{h}$ and $60 \mathrm{~km} / \mathrm{h}$ were examined, substituting the $50 \mathrm{~km} / \mathrm{h}$ and $70 \mathrm{~km} / \mathrm{h}$ initially assumed. As expected, a drop in the speed limits results in the TNTT and the OD pair accessibility increase; the opposite is valid for higher speed limits. For the combination of $60 \mathrm{~km} / \mathrm{h}$ and $80 \mathrm{~km} / \mathrm{h}$, the average OF value is $(-0.534154)$, while the respective value for the combination of 40 $\mathrm{km} / \mathrm{h}$ and $60 \mathrm{~km} / \mathrm{h}$ is $(-0.430380)$. The low coefficients of variation (5.08\% and $8.54 \%$, respectively) prove the algorithm's ability to produce consistent results.

\section{RESULTS AND DISCUSSION}

Comparison of the optimized network with either the intact network or its non-optimized, post-disaster form is not possible in terms of OF values. This is because the TNTT, the TNF and the special OD pair accessibility terms do not participate in the final OF value 
with their true values, as these are obtained from the optimization process, but with their normalized values. Also, when compared to the base case, the adjustment of the demand through the $\varphi^{r s}$ decision variables of the TNF term cannot lead to a safe conclusion as for the success (or not) of the demand regulation strategy. This is due to the nature of the strategy; demand regulation aims at the maximization of the TNF under the condition that the flow will not overwhelm the damaged transportation infrastructures. This implies that the demand allowed to travel on the post-disaster network will most likely be lower than the demand of the original network. Therefore, in a strict sense, any demand regulation strategy will provide results inferior to the base case and thus a comparison in that case is pointless. In terms of the TNTT, the optimized network yielded an improvement of $85.95 \%$ on average and $89.77 \%$ for the best run experiment, where $\mathrm{POP}=100$, $\mathrm{CR}=0.8, \mathrm{MR}=0.15$, when compared to the non-optimized network of the post-disaster phase. Finally, the comparison of the special OD pair accessibility values between the optimized and the non-optimized post-disaster networks indicated almost equivalent improvements of $79.8 \%$ on average, and $80 \%$ for $\mathrm{POP}=100$, $\mathrm{CR}=0.8, \mathrm{MR}=0.15$. The results indicate the computational efficiency of the algorithm in enhancing transportation network performance.

In addition to the $2 \mathrm{~h}$ experiments, each combination was also tested for running times of $1 \mathrm{~h}, 30$ $\mathrm{min}, 10 \mathrm{~min}$ and $5 \mathrm{~min}$. Table 3 summarizes the results in terms of OF value minimization. As expected, as the running time increases, the algorithm produces results of improved quality. The average OF value for the $1 \mathrm{~h}$ experiments is -0.476769 and for the 30 min experiments is -0.320793 , and thus inferior to the average OF value of the $2 \mathrm{~h}$ experiments by $5.77 \%$ and $57.73 \%$, and to the best experiment by $14.15 \%$ and
$73.12 \%$, respectively. The lower running times exhibit even greater differences from the values achieved in the $2 \mathrm{~h}$ experiments because the algorithm does not have enough time to efficiently search the search space. It can therefore be concluded that one hour is a sufficient running time to get a satisfactory solution to the problem.

\section{CONCLUSION}

Despite the challenging external conditions and their own vulnerability to structural and functional degradation, transportation networks are expected to remain adequately functional in the aftermath of a disaster in order to provide physical access to the affected communities as well as emergency services on the basis of the generated needs. It is also acknowledged that under the conditions of reduced serviceability, the damaged transportation infrastructures can benefit from the implementation of appropriate management strategies. In this context, this study examined the planning of operations on a post-disaster network with the use of an integrated model. Lane reversal, demand regulation and path activation were employed to provide an optimally reconfigured network with reallocated demand so that the network performance was maximized. Three performance indices were used for that purpose: the TNTT, the TNF and the special OD pair accessibility. The problem was formulated as a bi-level optimization model, with the upper level corresponding to the objective of performance maximization, while the lower level assigned traffic on the network on the basis of UE. A GA coupled with a traffic assignment process was used as a solution methodology. Application of the model on a real urban network under the scenario of a life-threatening, hazardous event on one of the network's nodes, proved the efficiency of the

Table 3 - Best results for objective function minimization for different running times

\begin{tabular}{|c|c|c|c|c|c|c|}
\hline & & & \multicolumn{4}{|c|}{ Objective function best runs } \\
\hline & & & $1 \mathrm{~h}$ trials & 30 min trials & 10 min trials & 5 min trials \\
\hline \multicolumn{7}{|c|}{ Experiment combination } \\
\hline POP & $\mathrm{CR}$ & MR & & & & \\
\hline 100 & 0.4 & 0.05 & -0.490817 & -0.277379 & 0.266934 & 1.167996 \\
\hline 100 & 0.4 & 0.10 & -0.503647 & -0.221063 & 0.175033 & 1.049697 \\
\hline 100 & 0.4 & 0.15 & -0.476631 & -0.322488 & 0.252707 & 0.942208 \\
\hline 100 & 0.6 & 0.05 & -0.492759 & -0.332508 & 0.207027 & 1.277866 \\
\hline 100 & 0.6 & 0.10 & -0.519013 & -0.309550 & 0.218749 & 1.001107 \\
\hline 100 & 0.6 & 0.15 & -0.446010 & -0.379549 & 0.185686 & 1.082943 \\
\hline 100 & 0.8 & 0.05 & -0.475042 & -0.372559 & 0.232834 & 1.217377 \\
\hline 100 & 0.8 & 0.10 & -0.449525 & -0.327775 & 0.215593 & 1.450128 \\
\hline 100 & 0.8 & 0.15 & -0.437475 & -0.344265 & 0.278121 & 1.581747 \\
\hline \multicolumn{3}{|c|}{ Average } & -0.476769 & -0.320793 & 0.225854 & 1.196785 \\
\hline
\end{tabular}


algorithm in enhancing network performance; the TNTT and the special OD pair accessibility terms showed significant improvements in their values when compared to the non-optimized, post-disaster network. More specifically, the algorithm achieved an improvement of $85.95 \%$ on average and $89.77 \%$ for the best run in terms of the TNTT, as well as improvements of $79.8 \%$ on average and $80 \%$ for the best run in terms of the special OD pair accessibility. The results indicate the computational efficiency of the algorithm in producing robust results of enhanced network performance and thus its value as an operation planning tool.

\section{ACKNOWLEDGMENTS}

This work is a part of the research financed by the Hellenic State Scholarships Foundation (IKY) and Siemens through the "Research Projects for Excellence IKY / Siemens" Grant, in the framework of the Hellenic Republic - Siemens Settlement Agreement.

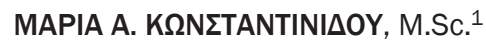

E-mail: mkonstaa@central.ntua.gr

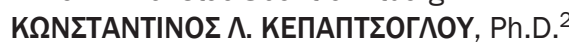

E-mail: kkepap@central.ntua.gr

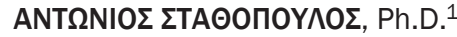

E-mail: a.stath@transport.ntua.gr

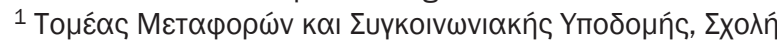

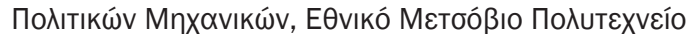

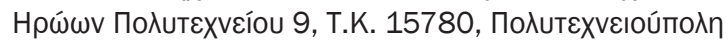

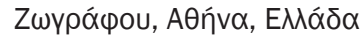

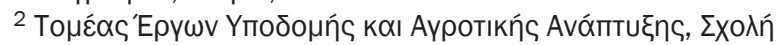

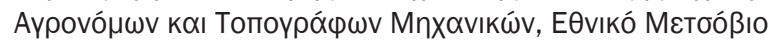

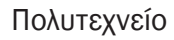

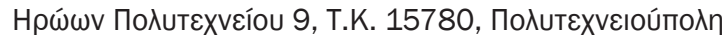

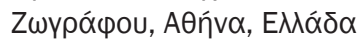

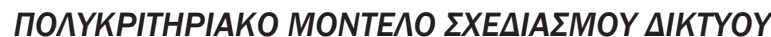

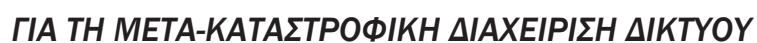 METAФOPSN}

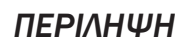

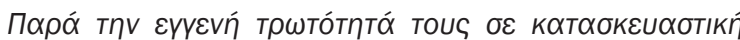

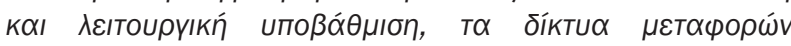

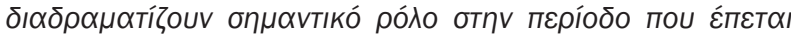

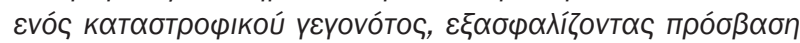

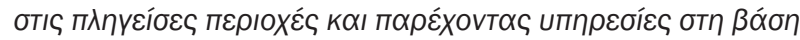

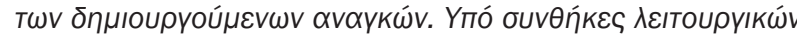

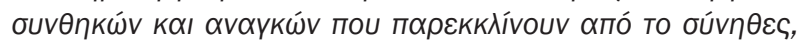

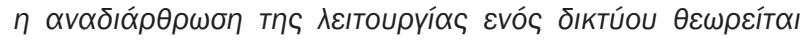

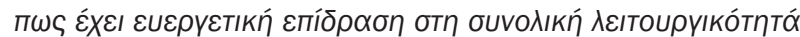

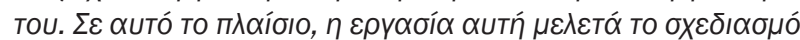

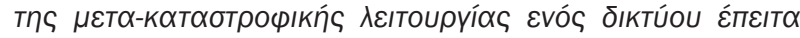

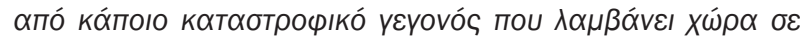

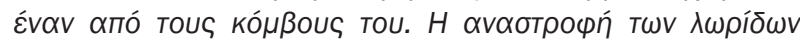

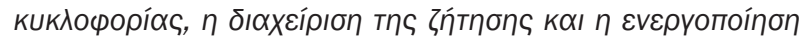

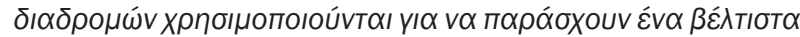

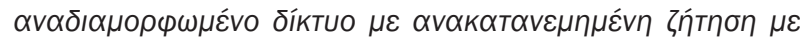

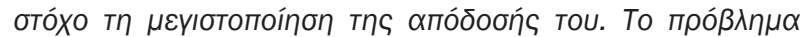

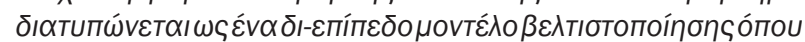

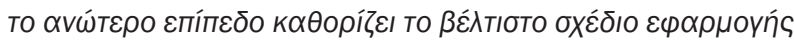

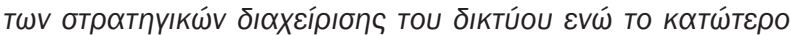

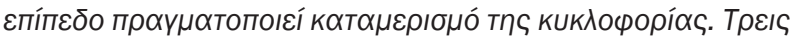

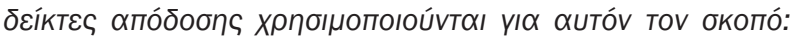

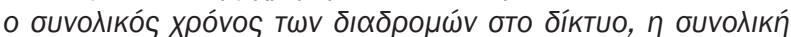

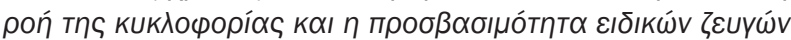

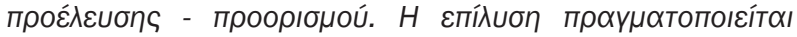

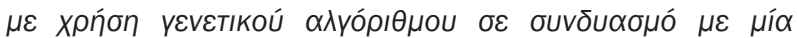

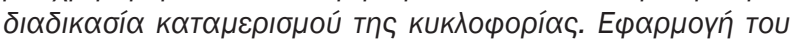

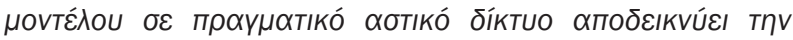

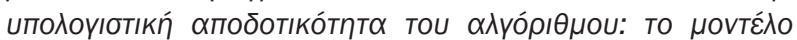

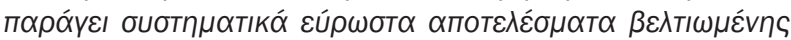

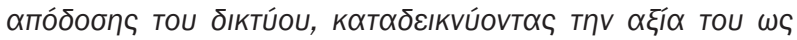

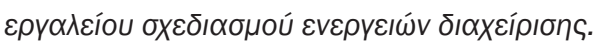

\section{$\Lambda E \equiv E I \Sigma$ K^EIDIA}

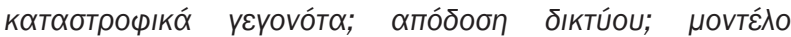

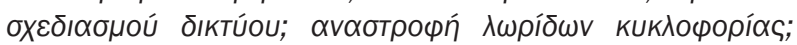

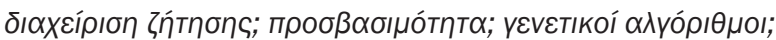

\section{REFERENCES}

[1] International Bank for Reconstruction and Development / The World Bank. Building resilience. Integrating climate and disaster risk into development. The World Bank Group experience. Washington DC: International Bank for Reconstruction and Development / The World Bank; 2013.

[2] Chen X, Kwan MP, Li Q, Chen J. A model for evacuation risk assessment with consideration of pre- and post-disaster factors. Computers, Environment and Urban Systems. 2012;36(3): 207-217.

[3] Barrett B, Ran B, Pillai R. Developing a dynamic traffic management modeling framework for hurricane evacuation. Transportation Research Record: Journal of the Transportation Research Board. 2000;1733: 115-121.

[4] Hamza-Lup GL, Hua KA, Le M, Peng R. Dynamic plan generation and real-time management techniques for traffic evacuation. IEEE Transactions on Intelligent Transportation Systems. 2008;9(4): 615-624.

[5] Konstantinidou MA, Kepaptsoglou KL, Karlaftis MG. Transportation network post-disaster planning and management: A review. Part I: Post-disaster transportation network performance. International Journal of Transportation. 2014;2(3); 1-16.

[6] Zimmerman C, Brodesky R, Karp J. Routes to effective evacuation planning primer series: Using highways for no-notice evacuations. Washington DC: FHWA, U.S. Department of Transportation. Report No. FHWAHOP-08-003, 2007.

[7] Menoni S. Chains of damages and failures in a metropolitan environment: Some observations on the Kobe earthquake in 1995. Journal of Hazardous Materials. 2001;86(1-3): 101-119.

[8] Nojima N. Prioritization in upgrading seismic performance of road network based on system reliability analysis. Paper presented at: The $3^{\text {rd }}$ China-Japan-US Trilateral Symposium on Lifeline Earthquake Engineering. Kunming, China; 1998.

[9] Chang SE, Nojima N. Measuring post-disaster transportation system performance: The 1995 Kobe earthquake in comparative perspective. Transportation Research Part A: Policy and Practice. 2001;35(6): 475-494.

[10] Chang SE. Transportation planning for disasters: An 
accessibility approach. Environment and Planning $A$. 2003;35(6): 1051-1072.

[11] Ukkusuri SV, Yushimito WF. A methodology to assess the criticality of highway transportation networks. Journal of Transportation Security. 2009;2(1-2): 29-46.

[12] lida $Y$, Kurauchi $F$, Shimada H. Traffic management system against major earthquakes. IATSS Research. 2000;24(2): 6-17.

[13] Lin DY, Eluru N, Waller TS, Bhat CR. Evacuation planning using the integrated system of activity-based modeling and dynamic traffic assignment. Transportation Research Record: Journal of the Transportation Research Board. 2009;2132: 69-77.

[14] Chiu YC, Zheng H, Villalobos J, Gautam B. Modeling no-notice mass evacuation using a dynamic traffic flow optimization model. IIE Transactions. 2007;39(1): 83-94.

[15] Liu HX, Ban JX, Ma W, Mirchandani PB. Model reference adaptive control framework for real-time traffic management under emergency evacuation. Journal of Urban Planning and Development. 2007;133(1): 43-50.

[16] Kepaptsoglou KL, Konstantinidou MA, Karlaftis MG, Stathopoulos A. Planning post-disaster operations in a highway network: Network design model with interdependencies. Transportation Research Record: Journal of the Transportation Research Board. 2014;2459; 1-10.

[17] Konstantinidou MA, Kepaptsoglou, KL, Karlaftis MG, Stathopoulos A. Joint evacuation and emergency traffic management model with consideration of emergency response needs. Transportation Research Record: Journal of the Transportation Research Board. 2015;2532; 107-117.

[18] Niemeier DA. Accessibility: An evaluation using consumer welfare. Transportation. 1997;24: 377-396.

[19] Bhat C, Handy S, Kockelman K, Mahmassani H, Chen Q, Weston L. Urban accessibility index: Literature review. Austin: Center for Transportation Research, The University of Texas at Austin. Report No. TX-01/74938-1, 2000.

[20] Geurs KT, Ritsema van Eck JR. Accessibility Measures: Review and Applications. Bilthoven: National Institute of Public Health and the Environment. RIVM Report 408505006, 2001.

[21] Scheurer J, Curtis C. Accessibility measures: Overview and practical applications. Working Paper No. 4: Accessibility measures. Australia: Curtin University of Technology; 2007.

[22] Taylor MAP, Susilawati. Remoteness and accessibility in the vulnerability analysis of regional road networks. Transportation Research Part A: Policy and Practice. 2012;46(5): 761-771.

[23] Bono F, Gutiérrez E. A network-based analysis of the impact of structural damage on urban accessibility following a disaster: The case of the seismically damaged Port Au Prince and Carrefour urban road networks. Journal of Transport Geography. 2011;19(6): 1443-1455.

[24] Chen A, Yang C, Kongsomsaksakul S, Lee M. Network-based accessibility measures for vulnerability analysis of degradable transportation networks. Networks and Spatial Economics. 2007;7(3): 241-256.

[25] Kondo R, Shiomi Y, Uno N. Network evaluation based on connectivity reliability and accessibility. Proceedings of the $4^{\text {th }}$ International Symposium on Transportation Network Reliability; 2010 July 22-23; University of Minnesota, US. New York: Springer; 2012.
[26] Sohn J. Evaluating the significance of highway network links under the flood damage: An accessibility approach. Transportation Research Part A: Policy and Practice. 2006;40(6): 491-506.

[27] Taylor, MAP, Sekhar SVC, D'Este GM. Application of accessibility based methods for vulnerability analysis of strategic road networks. Networks and Spatial Economics. 2006;6(3-4): 267-291.

[28] Taylor MAP, D' Este GM. Transport network vulnerability: A method for diagnosis of critical locations in transport infrastructure systems. In: Murray AT, Grubesic TH (editors). Critical infrastructure. Advances in spatial science. Berlin, Heidelberg: Springer; 2007. p. 9-30.

[29] Sumalee A, Kurauchi F. Network capacity reliability analysis considering traffic regulation after a major disaster. Networks and Spatial Economics. 2006;6(3-4): 205-219.

[30] Daganzo CF, So SK. Managing evacuation networks. Procedia - Social and Behavioral Sciences. 2011;17: 405-415.

[31] Sisiopiku VP. Application of traffic simulation modeling for improved emergency preparedness planning. Journal of Urban Planning and Development. 2007;133(1): 51-60.

[32] Chen M, Chen L, Miller-Hooks E. Traffic signal timing for urban evacuation. Journal of Urban Planning and Development. 2007;133(1): 30-42.

[33] Dixit VV, Radwan E. Hurricane evacuation: Origin, route, and destination. Journal of Transportation Safety \& Security. 2009;1(1): 74-84.

[34] Bretschneider S, Kimms A. Pattern-based evacuation planning for urban areas. European Journal of Operational Research. 2012;216(1): 57-69.

[35] Xie C, Turnquist MA. Lane-based evacuation network optimization: An integrated lagrangian relaxation and tabu search approach. Transportation Research Part C: Emerging Technologies. 2011;19(1): 40-63.

[36] Peeta S, Salman SF, Gunnec D, Viswanath K. Pre-disaster investment decisions for strengthening a highway network. Computers \& Operations Research. 2010;37(10): 1708-1719.

[37] Proos KA, Steven GP, Querin OM, Xie YM. Multicriterion evolutionary structural optimization using the weighted and the global criterion methods. AIAA Journal. 2001;39(10): 2006-2012.

[38] Farahani RZ, Miandoabchi E, Szeto WY, Rashidi H. A review of urban transportation network design problems. European Journal of Operational Research. 2013;229(2): 281-302.

[39] Xie, C, Lin, DY, Waller, TS. A dynamic evacuation network optimization problem with lane reversal and crossing elimination strategies. Transportation Research Part E: Logistics and Transportation Review. 2010;46(3): 295-316.

[40] Palisade Corporation. Guide to Using Evolver - The Genetic Algorithm Solver for Microsoft Excel. Version 5.0. New York: Palisade Corporation; 2010.

[41] Available from: https://www.tutorialspoint.com/genetic_algorithms/genetic_algorithms_crossover.htm

[42] Available from: https://www.tutorialspoint.com/genetic_algorithms/genetic_algorithms_mutation.htm 